\title{
FIELDS OF MODULI OF HYPERELLIPTIC CURVES
}

\author{
Bonnie HugGins
}

\begin{abstract}
Let $X$ be a hyperelliptic curve defined over a field $K$ of characteristic not equal to 2 . Let $\iota$ be the hyperelliptic involution of $X$. We show that $X$ can be defined over its field of moduli if Aut $(X) /\langle\iota\rangle$ is not cyclic. We construct explicit examples of hyperelliptic curves not definable over their field of moduli when $\operatorname{Aut}(X) /\langle\iota\rangle$ is cyclic.
\end{abstract}

\section{Introduction}

Let $X$ be a curve of genus $g$ defined over a field $K$ and let $K_{X}$ be the field of moduli of $X$. (See Section 2 for the definition of "field of moduli".) It is well known that if $g$ is 0 or 1 then $X$ admits a model defined over $K_{X}$. It is also well known that if the group of automorphisms of $X$ is trivial then $X$ can be defined over $K_{X}$. However, if $g \geq 2$ and $|\operatorname{Aut}(X)|>1$, the curve $X$ may not be definable over its field of moduli.

We examine the case where $X$ is hyperelliptic and $K$ is a field of characteristic not equal to 2. (For a similar examination in the case where $X$ is a smooth plane curve, see [8].) In this case $\operatorname{Aut}(X)$ is always nontrivial since it contains the hyperelliptic involution $\iota$. Examples of hyperelliptic curves not definable over their field of moduli are given on page 177 in [10]. In [6] it is shown that $X$ can be defined over $K_{X}$ if $g=2$ and $|\operatorname{Aut}(X)|>2$. In Theorem 4.2 and Corollary 4.4 of [9] it is shown that $X$ is definable over $K_{X}$ if $\operatorname{Char}(K)=0, g \geq 2$, and $\operatorname{Aut}(X) /\langle\iota\rangle$ has at least two involutions. In Section 1 of [9] and more recently in Section 4 of [7], it is conjectured that $X$ is definable over $K_{X}$ if $\operatorname{Char}(K)=0$ and $|\operatorname{Aut}(X)|>2$. The authors of [3] have attempted to classify all hyperelliptic curves over $\mathbb{C}$ with fields of moduli contained in $\mathbb{R}$ relative to $\mathbb{C} / \mathbb{R}$ but not definable over $\mathbb{R}$. Due to errors in their paper, some curves are missing from their list and many curves on their list are, in fact, definable over $\mathbb{R}$. In Section 6.2 , we give new examples of hyperelliptic $\mathbb{C}$-curves not definable over their fields of moduli relative to $\mathbb{C} / \mathbb{R}$. Each curve $X$ in has $\operatorname{Aut}(X) /\langle\iota\rangle$ cyclic of order $n$ for some $n>1$.

\section{Fields of moduli and fields of definition}

Definition 2.1. Let $K$ be a field. A variety over $K$ ( $K$-variety) is an integral separated scheme of finite type over $\operatorname{Spec} K$.

Notation 2.2. Let $K$ be a field, let $X$ be a $K$-variety, and let $F$ be an extension field of $K$. Let $X_{F}$ denote the base extension $X \times \times_{\operatorname{Spec} K} \operatorname{Spec} F$.

Definition 2.3. Let $K$ be a field. A curve over $K$ is a smooth, projective, geometrically integral $K$-variety of dimension 1 .

Received by the editors May 5, 2006. 
Definition 2.4. Let $K \subseteq F \subseteq \bar{F}$ be fields where $\bar{F}$ is an algebraic closure of $F$. Let $X$ be an $F$-variety. Then $X$ is defined over $K$ if and only if there is a $K$-variety $X^{\prime}$ such that $X_{F}^{\prime}$ is isomorphic (as an $F$-variety) to $X$. We say that $K$ is a field of definition of $X$. We say that $X$ is definable over $K$ if there is a $K$-variety $X^{\prime}$ such that $X_{\bar{F}}^{\prime}$ is isomorphic to $X_{\bar{F}}$.

Definition 2.5. Let $X$ be a curve over a field $K$. Let $\bar{K}$ be an algebraic closure of $K$. The field of moduli $K_{X}$ of $X$ is the intersection over all fields of definition of $X_{\bar{K}}$.

Due to Theorem 2.7 below, we may utilize an alternate definition of "field of moduli" that is defined relative to a given Galois extension.

Definition 2.6. Let $X$ be a curve over a field $F$ and let $K$ be a subfield of $F$ such that $F / K$ is Galois. The field of moduli of $X$ relative to the extension $F / K$ is defined as the fixed field $F^{H}$ of

$$
H:=\left\{\sigma \in \operatorname{Gal}(F / K) \mid X \cong{ }^{\sigma} X \text { over } F\right\} .
$$

Theorem 2.7. Let $X$ be a curve over a field $K$ and let $K_{X}$ be the field of moduli of $X$. Then $X$ is definable over $K_{X}$ if and only if given any algebraically closed field $F \supseteq K$, and any subfield $L \subseteq F$ with $F / L$ Galois, $X_{F}$ can be defined over its field of moduli relative to the extension $F / L$.

Proof. See Theorem 1.6.9 on page 12 of [8].

We have the following useful results.

Proposition 2.8. Let $X$ be a curve over a field $F$, let $K$ be a subfield of $F$ such that $F / K$ is Galois, let

$$
H:=\left\{\sigma \in \operatorname{Gal}(F / K) \mid X \cong{ }^{\sigma} X \text { over } F\right\},
$$

and let $K_{m}$ be the field of moduli of $X$ relative to $F / K$. Then the subgroup $H$ is a closed subgroup of $\operatorname{Gal}(F / K)$ for the Krull topology. That is,

$$
H=\operatorname{Gal}\left(F / K_{m}\right) .
$$

The field of $K_{m}$ is contained in each field of definition between $K$ and $F$ (in particular, $K_{m}$ is a finite extension of $K$ ). Hence if the field of moduli is a field of definition, it is the smallest field of definition between $F$ and $K$. Finally, the field of moduli of $X$ relative to the extension $F / K_{m}$ is $K_{m}$.

Proof. See Proposition 2.1 in [5].

Theorem 2.9 (Weil). Let $X$ be a curve over a field $F$ and let $K$ be a subfield of $F$ such that $F / K$ is Galois. Let $\Gamma=\operatorname{Gal}(F / K)$ and suppose for all $\sigma \in \Gamma$ there exists an $F$-isomorphism $f_{\sigma}: X \rightarrow{ }^{\sigma} X$ such that

$$
f_{\tau}^{\sigma} f_{\sigma}=f_{\sigma \tau}, \text { for all } \sigma, \tau \in \Gamma .
$$

Then there exist a $K$-curve $X^{\prime}$ and an isomorphism

$$
f: X \rightarrow X_{F}^{\prime}
$$

defined over $F$ such that

$$
f_{\sigma}=\left(f^{-1}\right)^{\sigma} f, \text { for all } \sigma \in \Gamma \text {. }
$$


Proof. See the proof of Theorem 1 of [13].

The following three results of Dèbes, Emsalem, and Douai will be of use to us. They rely on the notions of a cover and the field of moduli of a cover, for which we refer the reader to $\S 2.4$ in [4].

Theorem 2.10. Let $F / K$ be a Galois extension and $X$ be a curve of genus larger than 1 defined over $F$ with $K$ as field of moduli. Then there exists a $K$-model $B$ of the curve $X / \operatorname{Aut}(X)$ such that the cover $X \rightarrow B_{F}$ with $K$-base $B$ is of field of moduli $K$.

Proof. See Theorem 3.1 in [5]. The authors make the additional assumption that the characteristic of $K$ does not divide $|\operatorname{Aut}(X)|$ but do not use it in their proof.

Corollary 2.11. Suppose that $K$ is a finite field and that $F$ is algebraically closed. Then $X$ can be defined over $K$.

Proof. It suffices to show that the cover $X \rightarrow B_{F}$ with $K$-base $B$ can be defined over $K$, since a field of definition of the cover is automatically a field of definition of $X$. By Theorem 2.10, the field of moduli of the cover $X \rightarrow B_{F}$ with $K$-base $B$ is $K$. If $K$ is a finite field then $\operatorname{Gal}(F / K)$ is a projective profinite group. In this case, by Corollary 3.3 of [4] the cover $X \rightarrow B_{F}$ can be defined over $K$.

Corollary 2.12. Suppose that $F$ is algebraically closed and that $X$ is a hyperelliptic curve. If $B$ has a $K$-rational point, then $K$ is a field of definition of $X$.

Proof. It suffices to show that the cover $X \rightarrow B_{F}$ with $K$-base $B$ can be defined over $K$, since a field of definition of the cover is automatically a field of definition of $X$. By Theorem 2.10, the field of moduli of the cover $X \rightarrow B_{F}$ with $K$-base $B$ is $K$. By Corollary 2.11, we may assume that $K$ is infinite. Since $B \cong_{K} \mathbb{P}_{K}^{1}, B$ has a rational point off the branch point set of $X \rightarrow B_{F}$. Then by Corollary 3.4 and $\S 2.9$ of [4], the cover can be defined over $K$.

The curve $B$ of Theorem 2.10 and Corollary 2.12 is called the canonical model of $X / \operatorname{Aut}(X)$ over the field of moduli of $X$.

\section{Finite subgroups of the 2-dimensional projective general linear groups}

Throughout this section let $F$ be an algebraically closed field of characteristic $p$ with $p=0$ or $p>2$. We will use a matrix with round brackets to denote an element of $\mathrm{GL}_{n}(F)$ and a matrix with square brackets to denote the image in $\mathrm{PGL}_{n}(F)$ of an element of $\mathrm{GL}_{n}(F)$.

Lemma 3.1. Any finite subgroup $\mathfrak{G}$ of $\mathrm{PGL}_{2}(F)$ is conjugate to one of the following groups:

Case I: when $p=0$ or $|\mathfrak{G}|$ is relatively prime to $p$.

(a) $\mathfrak{G}_{C_{n}}:=\left\{\left[\begin{array}{cc}\zeta^{r} & 0 \\ 0 & 1\end{array}\right]: r=0,1, \ldots, n-1\right\} \cong C_{n}, n \geq 1$

(b) $\mathfrak{G}_{D_{2 n}}:=\left\{\left[\begin{array}{cc}\zeta^{r} & 0 \\ 0 & 1\end{array}\right],\left[\begin{array}{cc}0 & \zeta^{r} \\ 1 & 0\end{array}\right]: r=0,1, \ldots, n-1\right\} \cong D_{2 n}, n>1$ 
(c) $\mathfrak{G}_{A_{4}}:=\left\{\left[\begin{array}{cc} \pm 1 & 0 \\ 0 & 1\end{array}\right],\left[\begin{array}{cc}0 & \pm 1 \\ 1 & 0\end{array}\right],\left[\begin{array}{cc}i^{\nu} & i^{\nu} \\ 1 & -1\end{array}\right],\left[\begin{array}{cc}i^{\nu} & -i^{\nu} \\ 1 & 1\end{array}\right],\left[\begin{array}{cc}1 & i^{\nu} \\ 1 & -i^{\nu}\end{array}\right]\right.$,

$$
\left.\left[\begin{array}{cc}
-1 & -i^{\nu} \\
1 & -i^{\nu}
\end{array}\right]: \nu=1,3\right\} \cong A_{4}
$$

(d) $\mathfrak{G}_{S_{4}}:=\left\{\left[\begin{array}{cc}i^{\nu} & 0 \\ 0 & 1\end{array}\right],\left[\begin{array}{cc}0 & i^{\nu} \\ 1 & 0\end{array}\right],\left[\begin{array}{cc}i^{\nu} & -i^{\nu+\nu^{\prime}} \\ 1 & i^{\nu^{\prime}}\end{array}\right]: \nu, \nu^{\prime}=0,1,2,3\right\} \cong S_{4}$

(e) $\mathfrak{G}_{A_{5}}:=\left\{\left[\begin{array}{cc}\epsilon^{r} & 0 \\ 0 & 1\end{array}\right],\left[\begin{array}{cc}0 & \epsilon^{r} \\ -1 & 0\end{array}\right],\left[\begin{array}{cc}\epsilon^{r} \omega & \epsilon^{r-s} \\ 1 & -\epsilon^{-s} \omega\end{array}\right],\left[\begin{array}{cc}\epsilon^{r} \bar{\omega} & \epsilon^{r-s} \\ 1 & -\epsilon^{-s} \bar{\omega}\end{array}\right]\right.$ :

$$
r, s=0,1,2,3,4\} \cong A_{5}
$$

where $\omega:=\frac{-1+\sqrt{5}}{2}, \bar{\omega}:=\frac{-1-\sqrt{5}}{2}, \zeta$ is a primitive $n^{\text {th }}$ root of unity, $\epsilon$ is a primitive $5^{\text {th }}$ root of unity, and $i$ is a primitive $4^{\text {th }}$ root of unity.

Case II: when $|\mathfrak{G}|$ is divisible by $p$.

(f) $\mathfrak{G}_{\beta, A}:=\left\{\left[\begin{array}{cc}\beta^{k} & a \\ 0 & 1\end{array}\right]: a \in A, k \in \mathbb{Z}\right\}$, where $A$ is a finite additive subgroup of $F$ containing 1 and $\beta$ is a root of unity such that $\beta A=A$

(g) $\operatorname{PSL}_{2}\left(\mathbb{F}_{q}\right)$

(h) $\mathrm{PGL}_{2}\left(\mathbb{F}_{q}\right)$

where $\mathbb{F}_{q}$ is the finite field with $q:=p^{r}$ elements, where $r>0$.

Proof. See $\S \S 71-74$ in [12] and Chapter 3 in [11].

Remark 3.2. It can be directly verified that $\mathfrak{G}_{A_{4}}$ and $\mathfrak{G}_{S_{4}}$ are subgroups of $\mathrm{PGL}_{2}(F)$ when the characteristic of $F$ is 3 . Indeed, in this case $\mathfrak{G}_{A_{4}}$ is $\mathrm{PGL}_{2}(F)$ conjugate to $\mathrm{PSL}_{2}\left(\mathbb{F}_{3}\right)$ and $\mathfrak{G}_{S_{4}}$ is $\mathrm{PGL}_{2}(F)$ conjugate to $\mathrm{PGL}_{2}\left(\mathbb{F}_{3}\right)$. So the result of Lemma 3.3(b) is still valid in characteristic 3 .

Lemma 3.3. Let $N(\mathfrak{G})$ be the normalizer of $\mathfrak{G}$ in $\mathrm{PGL}_{2}(F)$. Then
(a) $N\left(\mathfrak{G}_{C_{n}}\right)=\left\{\left[\begin{array}{ll}\alpha & 0 \\ 0 & 1\end{array}\right],\left[\begin{array}{cc}0 & \alpha \\ 1 & 0\end{array}\right]: \alpha \in F^{\times}\right\}$if $n>1$,
(b) $N\left(\mathfrak{G}_{D_{4}}\right)=\mathfrak{G}_{S_{4}}, N\left(\mathfrak{G}_{D_{2 n}}\right)=\mathfrak{G}_{D_{4 n}}$ if $n>2$,
(c) $N\left(\mathfrak{G}_{A_{4}}\right)=\mathfrak{G}_{S_{4}}$,
(d) $N\left(\mathfrak{G}_{S_{4}}\right)=\mathfrak{G}_{S_{4}}$,
(e) $N\left(\mathfrak{G}_{A_{5}}\right)=\mathfrak{G}_{A_{5}}$,
(g) $N\left(\mathrm{PSL}_{2}\left(\mathbb{F}_{q}\right)\right)=\mathrm{PGL}_{2}\left(\mathbb{F}_{q}\right)$, and
(h) $N\left(\mathrm{PGL}_{2}\left(\mathbb{F}_{q}\right)\right)=\mathrm{PGL}_{2}\left(\mathbb{F}_{q}\right)$.

\section{Proof.}

(a) See $\S 71$ in [12].

(b) Since $\mathfrak{G}_{D_{4}}$ is a normal subgroup of $\mathfrak{G}_{S_{4}}, \mathfrak{G}_{S_{4}} \subseteq N\left(\mathfrak{G}_{D_{4}}\right)$. Conjugation of $\mathfrak{G}_{D_{4}}$ by $\mathfrak{G}_{S_{4}}$ gives a homomorphism $\mathfrak{G}_{S_{4}} \rightarrow \operatorname{Aut}\left(D_{4}\right) \cong S_{3}$. A computation shows that the centralizer $Z$ of $\mathfrak{G}_{D_{4}}$ in $\mathrm{PGL}_{2}(F)$ is $\mathfrak{G}_{D_{4}}$. The kernel of this homomorphism is $Z \cap \mathfrak{G}_{S_{4}}=Z$. Since $\mathfrak{G}_{S_{4}} / Z \cong S_{3}$, every automorphism of $\mathfrak{G}_{D_{4}}$ is given by conjugation by an element of $\mathfrak{G}_{S_{4}}$. Let $U \in N\left(\mathfrak{G}_{D_{4}}\right)$. Then $U V \in Z=\mathfrak{G}_{D_{4}}$ for some $V \in \mathfrak{G}_{S_{4}}$, so $U \in \mathfrak{G}_{S_{4}}$.

For $n>2$, see $\S 71$ in [12].

(c) Since $\mathfrak{G}_{D_{4}}$ is a characteristic subgroup of $\mathfrak{G}_{A_{4}}, N\left(\mathfrak{G}_{A_{4}}\right) \subseteq N\left(\mathfrak{G}_{D_{4}}\right)=\mathfrak{G}_{S_{4}}$. As $\mathfrak{G}_{A_{4}}$ is normal in $\mathfrak{G}_{S_{4}}$, we get $N\left(\mathfrak{G}_{A_{4}}\right)=\mathfrak{G}_{S_{4}}$. 
(d) Since $\mathfrak{G}_{A_{4}}$ is a characteristic subgroup of $\mathfrak{G}_{S_{4}}, N\left(\mathfrak{G}_{S_{4}}\right) \subseteq N\left(\mathfrak{G}_{A_{4}}\right)=\mathfrak{G}_{S_{4}}$. Thus $N\left(\mathfrak{G}_{S_{4}}\right)=\mathfrak{G}_{S_{4}}$.

(e) Conjugation of $\mathfrak{G}_{A_{5}}$ by $N\left(\mathfrak{G}_{A_{5}}\right)$ gives a homomorphism $N\left(\mathfrak{G}_{A_{5}}\right) \rightarrow \operatorname{Aut}\left(A_{5}\right)$. The kernel of this homomorphism is the centralizer of $\mathfrak{G}_{A_{5}}$ in $N\left(\mathfrak{G}_{A_{5}}\right)$, which is just the centralizer $Z$ of $\mathfrak{G}_{A_{5}}$ in $\mathrm{PGL}_{2}(F)$. A computation shows that $Z$ is just the identity. Since $\operatorname{Aut}\left(A_{5}\right)$ is finite, $N\left(\mathfrak{G}_{A_{5}}\right)$ is a finite subgroup of $\mathrm{PGL}_{2}(F)$. Since $\mathfrak{G}_{A_{5}} \subseteq N\left(\mathfrak{G}_{A_{5}}\right)$, by Lemma 3.1 we must have $N\left(\mathfrak{G}_{A_{5}}\right)=\mathfrak{G}_{A_{5}}$.

(g) We first show that $N\left(\mathrm{PSL}_{2}\left(\mathbb{F}_{q}\right)\right)$ is finite. Conjugation of $\mathrm{PSL}_{2}\left(\mathbb{F}_{q}\right)$ by $N\left(\mathrm{PSL}_{2}\left(\mathbb{F}_{q}\right)\right)$ gives a homomorphism $N\left(\mathrm{PSL}_{2}\left(\mathbb{F}_{q}\right)\right) \rightarrow \operatorname{Aut}\left(\mathrm{PSL}_{2}\left(\mathbb{F}_{q}\right)\right)$. The kernel of this homomorphism is the centralizer $Z$ of $\mathrm{PSL}_{2}\left(\mathbb{F}_{q}\right)$ in $\mathrm{PGL}_{2}(F)$. A computation shows that $Z$ is just the identity. Since $\operatorname{Aut}\left(\operatorname{PSL}_{2}\left(\mathbb{F}_{q}\right)\right)$ is finite, so is $N\left(\mathrm{PSL}_{2}\left(\mathbb{F}_{q}\right)\right)$. By Lemma 3.1 any finite subgroup of $\mathrm{PGL}_{2}(F)$ containing $\mathrm{PSL}_{2}\left(\mathbb{F}_{q}\right)$ must be isomorphic to either $\mathrm{PGL}_{2}\left(\mathbb{F}_{q^{\prime}}\right)$ or $\mathrm{PSL}_{2}\left(\mathbb{F}_{q^{\prime}}\right)$ for some $q^{\prime}$. Since $\mathrm{SL}_{2}\left(\mathbb{F}_{q}\right)$ is normal in $\mathrm{GL}_{2}\left(\mathbb{F}_{q}\right), \mathrm{PSL}_{2}\left(\mathbb{F}_{q}\right)$ is a normal subgroup of $\mathrm{PGL}_{2}\left(\mathbb{F}_{q}\right)$. So $\mathrm{PGL}_{2}\left(\mathbb{F}_{q}\right) \subseteq N\left(\mathrm{PSL}_{2}\left(\mathbb{F}_{q}\right)\right)$, in particular $\mathrm{PSL}_{2}\left(\mathbb{F}_{q}\right)$ is strictly contained in $N\left(\mathrm{PSL}_{2}\left(\mathbb{F}_{q}\right)\right)$. By the corollary on page 80 of [11], $\mathrm{PSL}_{2}\left(\mathbb{F}_{q^{\prime}}\right)$ is simple for $q^{\prime}>3$. It follows that $N\left(\mathrm{PSL}_{2}\left(\mathbb{F}_{q}\right)\right) \neq \mathrm{PSL}_{2}\left(\mathbb{F}_{q}\right)$ for $q \geq 3$. By Theorem 9.9 on page 78 of [11], the only nontrivial normal subgroup of $\mathrm{PGL}_{2}\left(\mathbb{F}_{q^{\prime}}\right)$ is $\mathrm{PSL}_{2}\left(\mathbb{F}_{q^{\prime}}\right)$ if $q^{\prime}>3$. Therefore $N\left(\mathrm{PSL}_{2}\left(\mathbb{F}_{q}\right)\right)=\mathrm{PGL}_{2}\left(\mathbb{F}_{q}\right)$.

(h) Clear from the proof of the previous case.

\section{Isomorphisms of hyperelliptic curves}

Throughout this section let $K$ be a perfect field of characteristic not equal to 2, let $F$ be an algebraic closure of $K$, and let $X$ be a hyperelliptic curve over $F$. In particular, $X$ admits a degree-2 morphism to $\mathbb{P}_{F}^{1}$ and the genus of $X$ is at least 2. Each element of $\operatorname{Aut}(X)$ induces an automorphism of $\mathbb{P}_{F}^{1}$ fixing the branch points. The number of branch points is $\geq 3$ (in fact $\geq 6$ ), so $\operatorname{Aut}(X)$ is finite. We get a homomorphism $\operatorname{Aut}(X) \rightarrow \operatorname{Aut}\left(\mathbb{P}_{F}^{1}\right)=\mathrm{PGL}_{2}(F)$ with kernel generated by the hyperelliptic involution $\iota$. Let $\mathfrak{G} \subset \mathrm{PGL}_{2}(F)$ be the image of this homomorphism. Replacing the original map $X \rightarrow \mathbb{P}_{F}^{1}$ by its composition with an automorphism $g \in \operatorname{Aut}\left(\mathbb{P}_{F}^{1}\right)=\mathrm{PGL}_{2}(F)$ has the effect of changing $\mathfrak{G}$ to $g \mathfrak{G} g^{-1}$, so we may assume that $\mathfrak{G}$ is one of the groups listed in Lemma 3.1. Fix an equation $y^{2}=f(x)$ for $X$ where $f \in F[x]$ and $\operatorname{disc}(f) \neq 0$. So the function field $F(X)$ equals $F(x, y)$.

Proposition 4.1. Let $X^{\prime}$ be a hyperelliptic curve over $F$ given by $y^{2}=f^{\prime}(x)$, where $f^{\prime}(x)$ is another squarefree polynomial in $F[x]$. Every isomorphism $\varphi: X \rightarrow X^{\prime}$ is given by an expression of the form:

$$
(x, y) \mapsto\left(\frac{a x+b}{c x+d}, \frac{e y}{(c x+d)^{g+1}}\right),
$$

for some $M=\left(\begin{array}{ll}a & b \\ c & d\end{array}\right) \in \mathrm{GL}_{2}(F)$ and $e \in F^{\times}$. The pair $(M, e)$ is unique up to replacement by $\left(\lambda M, e \lambda^{g+1}\right)$ for $\lambda \in F^{\times}$. If $\varphi^{\prime}: X^{\prime} \rightarrow X^{\prime \prime}$ is another isomorphism, given by $\left(M^{\prime}, e^{\prime}\right)$, then the composition $\varphi^{\prime} \varphi$ is given by $\left(M^{\prime} M, e^{\prime} e\right)$.

Proof. See Proposition 2.1 in [1]. 
Throughout the rest of this section assume that $K$ is the field of moduli of $X$ relative to the extension $F / K$ and let $\Gamma=\operatorname{Gal}(F / K)$.

Lemma 4.2. Suppose $\sigma \in \Gamma$ and suppose that the isomorphism $\varphi: X \rightarrow{ }^{\sigma} X$ is given by $(M, e)$. Let $\bar{M}$ be the image of $M$ in $\mathrm{PGL}_{2}(F)$. If $\mathfrak{G} \neq \mathfrak{G}_{\beta, A}$ then $\bar{M}$ is in the normalizer $N(\mathfrak{G})$ of $\mathfrak{G}$ in $\mathrm{PGL}_{2}(F)$. If $\mathfrak{G}=\mathfrak{G}_{\beta, A}$ then $M$ is an upper triangular matrix.

Proof. Since $\operatorname{Aut}\left({ }^{\sigma} X\right)=\left\{\psi^{\sigma} \mid \psi \in \operatorname{Aut}(X)\right\}$, the group of automorphisms of $\mathbb{P}^{1}$ induced by $\operatorname{Aut}\left({ }^{\sigma} X\right)$ is $\mathfrak{G}^{\sigma}:=\left\{U^{\sigma} \mid U \in \mathfrak{G}\right\}$.

Let $\psi$ be an automorphism of $X$ given by $(V, v)$. Since $\psi$ is an automorphism, $V \in \mathrm{GL}_{2}(F)$ is a lift of some element $\bar{V} \in \mathfrak{G}$. Then $\varphi \psi \varphi^{-1}$ is an automorphism of ${ }^{\sigma} X$ given by $\left(M V M^{-1}, v\right)$. We have $\overline{M V M^{-1}}=\bar{M} \bar{V} \bar{M}^{-1} \in \mathfrak{G}^{\sigma}$. It follows that $\bar{M} \mathfrak{G} \bar{M}^{-1}=\mathfrak{G}^{\sigma}$. If $\mathfrak{G} \neq \mathfrak{G}_{\beta, A}$, by Lemma $3.1, \mathfrak{G}^{\sigma}=\mathfrak{G}$. So $\bar{M} \in N(\mathfrak{G})$. If $\mathfrak{G}=\mathfrak{G}_{\beta, A}$, then since $\mathfrak{G}^{\sigma}$ has an elementary abelian subgroup of the same form as $\mathfrak{G}$, a simple computation shows that $M$ is an upper triangular matrix.

Lemma 4.3. Suppose that for every $\tau \in \Gamma$ there exists an isomorphism $\varphi_{\tau}: X \rightarrow{ }^{\tau} X$ given by $\left(M_{\tau}, e\right)$ where $\bar{M}_{\tau} \in \mathfrak{G}^{\tau}$. Then $X$ can be defined over $K$. Furthermore, $X$ is given by an equation of the form $z^{2}=h(x)$ where $h \in K[x]$.

Proof. Let $P_{1}, \ldots, P_{n}$ be the hyperelliptic branch points of $X \rightarrow \mathbb{P}^{1}$. Let $\tau \in \Gamma$. The isomorphism $\varphi_{\tau}: X \rightarrow{ }^{\tau} X$ induces an isomorphism on the canonical images $\mathbb{P}^{1} \rightarrow \mathbb{P}^{1}$ which is given by $\bar{M}_{\tau}$. Write $\tau(\infty)=\infty$. The hypothesis $\bar{M}_{\tau} \in \mathfrak{G}^{\tau}$ implies that $\bar{M}_{\tau}$ maps $\left\{\tau\left(P_{1}\right), \ldots, \tau\left(P_{n}\right)\right\}$ to itself; since it also maps $\left\{P_{1}, \ldots, P_{n}\right\}$ to $\left\{\tau\left(P_{1}\right), \ldots, \tau\left(P_{n}\right)\right\}$, we get $\left\{\tau\left(P_{1}\right), \ldots, \tau\left(P_{n}\right)\right\}=\left\{P_{1}, \ldots, P_{n}\right\}$. So

$$
h(x):=\prod_{P_{j} \neq \infty}\left(x-P_{j}\right) \in K[x] .
$$

It follows that $X$ can be defined over $K$.

Corollary 4.4. Suppose that $N(\mathfrak{G})=\mathfrak{G}$ and $\mathfrak{G} \neq \mathfrak{G}_{\beta, A}$. Then $X$ can be defined over $K$.

Proof. By Lemma 3.1, $\mathfrak{G}^{\sigma}=\mathfrak{G}$ for all $\sigma \in \Gamma$. Let $\tau \in \Gamma$. By Lemma 4.2, any isomorphism $X \rightarrow{ }^{\tau} X$ is given by $(M, e)$ where $\bar{M} \in N(\mathfrak{G})=\mathfrak{G}=\mathfrak{G}^{\tau}$.

\section{The main result}

Let $K$ be a perfect field, let $F$ be an algebraic closure of $K$, and let $\Gamma=\operatorname{Gal}(F / K)$. Let $X$ be a hyperelliptic curve over $F$ and let $B$ be the canonical $K$-model of $X / \operatorname{Aut}(X)$ given in Theorem 2.10. In the proof of Theorem 2.10, Dèbes and Emsalem show the canonical model exists by using the following argument. For all $\sigma \in \Gamma$ there exists an isomorphism $\varphi_{\sigma}: X \rightarrow{ }^{\sigma} X$ defined over $F$. Each induces an isomorphism $\tilde{\varphi_{\sigma}}: X / \operatorname{Aut}(X) \rightarrow{ }^{\sigma} X / \operatorname{Aut}\left({ }^{\sigma} X\right)$ that makes the following diagram commute:

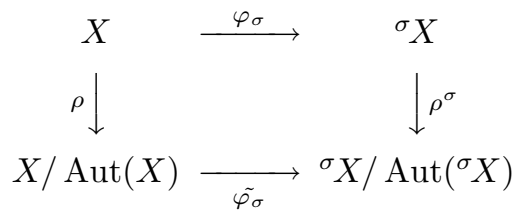


Composing $\tilde{\varphi_{\sigma}}$ with the canonical isomorphism

$$
i_{\sigma}:{ }^{\sigma} X / \operatorname{Aut}\left({ }^{\sigma} X\right) \rightarrow{ }^{\sigma}(X / \operatorname{Aut}(X))
$$

we obtain an isomorphism

$$
\overline{\varphi_{\sigma}}: X / \operatorname{Aut}(X) \rightarrow{ }^{\sigma}(X / \operatorname{Aut}(X))
$$

The family $\left\{\overline{\varphi_{\tau}}\right\}_{\tau \in \Gamma}$ satisfy Weil's cocycle condition $\bar{\varphi}_{\tau}^{\sigma} \overline{\varphi_{\sigma}}=\overline{\varphi_{\sigma \tau}}$ given in Theorem 2.9. This shows that $B$ exists.

Let $F\left(B_{F}\right)$ be the function field of $B_{F}$. Since $B_{F} \cong \mathbb{P}^{1}, F\left(B_{F}\right)=F(t)$ for some element $t$. We use $t$ as a coordinate on $B_{F}$. Suppose $\sigma \in \Gamma$ and suppose that $\overline{\varphi_{\sigma}}$ is given by

$$
t \mapsto \frac{a t+b}{c t+d}
$$

Define $\sigma^{*} \in \operatorname{Aut}(F(t) / K)$ by

$$
\sigma^{*}(t)=\frac{a t+b}{c t+d}, \sigma^{*}(\alpha)=\sigma(\alpha), \alpha \in F .
$$

One can verify that $(\sigma \tau)^{*}(w)=\sigma^{*}\left(\tau^{*}(w)\right)$ for all $w \in F(t)$. So we get a homomorphism $\Gamma \rightarrow \operatorname{Aut}\left(F\left(B_{F}\right) / K\right), \sigma \mapsto \sigma^{*}$. The curve $B$ is the variety over $K$ corresponding to the fixed field of $\Gamma^{*}=\left\{\sigma^{*}\right\}_{\sigma \in \Gamma}$. The following lemma and corollary will be of use.

Lemma 5.1. Let $C$ be a curve of genus 0 over $K$ and suppose that $C$ has a divisor $D$ rational over $K$ of odd degree. Then $C(K) \neq \emptyset$.

Proof. Let $\omega$ be a canonical divisor on $C$. Since $\operatorname{deg}(\omega)=-2$, we can take a linear combination of $D$ and $\omega$ to obtain a divisor $D^{\prime}$ of degree 1 . Since $\operatorname{deg}\left(\omega-D^{\prime}\right)<0$, by the Riemann-Roch theorem $l\left(D^{\prime}\right)>0$. So there exists an effective divisor $D^{\prime \prime}$ linearly equivalent to $D^{\prime}$ rational over $K$. Since $D^{\prime \prime}$ is effective and of degree 1 it consists of a point in $C(K)$.

Corollary 5.2. Let $L / K$ be a separable field extension of odd degree. Let $C$ be a curve of genus 0 defined over $K$ and suppose that $C(L) \neq \emptyset$. Then $C(K) \neq \emptyset$.

Proof. Let $P \in C(L)$ and let $n=[L: K]$. Let $\tau_{1}, \ldots, \tau_{n}$ be the distinct embeddings of $L$ into an algebraic closure of $L$. Then $D=\Sigma \tau_{i}(P)$ is a divisor of degree $n$ defined over $K$. By Lemma 5.1, $C(K) \neq \emptyset$.

Theorem 5.3. Let $K$ be a perfect field of characteristic not equal to 2 and let $F$ be an algebraic closure of $K$. Let $X$ be a hyperelliptic curve over $F$ and let $\mathfrak{G}=\operatorname{Aut}(X) /\langle\iota\rangle$ where $\iota$ is the hyperelliptic involution of $X$. Suppose that $\mathfrak{G}$ is not cyclic or that $\mathfrak{G}$ is cyclic of order divisible by the characteristic of $F$. Then $X$ can be defined over its field of moduli relative to the extension $F / K$.

Proof. Let $\Gamma=\operatorname{Gal}(F / K)$. By Proposition 2.8 we may assume that $K$ is the field of moduli of $X$. By Proposition 4.1 we may assume that $\mathfrak{G}$ is given by one of the groups in Lemma 3.1. Fix an equation $y^{2}=f(x)$ for $X$ where $f \in F[x]$ and $\operatorname{disc}(f) \neq 0$. So the function field $F(X)$ equals $F(x, y)$. There are eight cases. 
(b1) $\mathfrak{G} \cong D_{4}$. The element $t:=x^{2}+x^{-2}$ is fixed by $\mathfrak{G}_{D_{4}}$ and is a rational function of degree 4 in $x$. So the function field of $X / \operatorname{Aut}(X)$ equals $F(t)$. We use $t$ as a coordinate on $X / \operatorname{Aut}(X)$. The map $\rho: X \rightarrow X / \operatorname{Aut}(X)$ is given by $(x, y) \mapsto\left(x^{2}+x^{-2}\right)$. Let $\sigma \in \Gamma$. By Lemmas 4.2 and $3.3, \varphi_{\sigma}: X \rightarrow{ }^{\sigma} X$ is given by $(M, e)$ where $\bar{M} \in \mathfrak{G}_{S_{4}}$. A computation shows that $\sigma^{*}(t)$ is one of the following:
i. $t$
ii. $-t$
iii. $\frac{2 t+12}{t-2}$
iv. $\frac{2 t-12}{-t-2}$
v. $\frac{2 t-12}{t+2}$
vi. $\frac{2 t+12}{-t+2}$.

Since $\bar{\varphi}_{\tau}: X / \operatorname{Aut}(X) \rightarrow{ }^{\tau}(X / \operatorname{Aut}(X))$ is defined over $K$ for all $\tau \in \Gamma$, we have $\overline{\varphi_{\tau}} \overline{\varphi_{\sigma}}=\overline{\varphi_{\sigma \tau}}$ for all $\tau \in \Gamma$. The fractional linear transformations i through vi form a group under composition isomorphic to $S_{3}$. The map $\left.\tau \mapsto \tau^{*}\right|_{K(t)}$ defines a homomorphism from $\Gamma$ to this group. The kernel of this homomorphism is $\Lambda:=\left\{\tau \in \Gamma \mid \tau^{*}(t)=t\right\}$. So $|\Gamma / \Lambda|=1,2,3$, or 6 .

Case 1: $|\Gamma / \Lambda|=1$. In this case the fixed field of $\Gamma^{*}$ is $K(t)$ and $B=\mathbb{P}_{K}^{1}$.

Case 2: $|\Gamma / \Lambda|=2$. Let $\sigma$ be a representative of the nontrivial coset. There are three cases.

(i) $\sigma^{*}(t)=-t$. Then $t=0$ corresponds to a point $P \in B(K)$.

(ii) $\sigma^{*}(t)=\frac{2 t+12}{t-2}$. Then $t=6$ corresponds to a point $P \in B(K)$.

(iii) $\sigma^{*}(t)=\frac{2 t-12}{-t-2}$. Then $t=-6$ corresponds to a point $P \in B(K)$.

Case 3: $|\Gamma / \Lambda|=3$. Since the fixed field of $\Lambda^{*}$ is $F^{\Lambda}(t), B$ has a $F^{\Lambda}$-rational point. By Corollary 5.2 , since $\left[F^{\Lambda}: K\right]$ is odd, $B$ has a $K$-rational point.

Case 4: $|\Gamma / \Lambda|=6$. Let $\Pi$ be a subgroup of $\Gamma$ containing $\Lambda$ such that $\Pi / \Lambda$ is a subgroup of $\Gamma / \Lambda$ of order 2 . By Case $2, B$ has a $F^{\Pi}$ rational point. Since $\left[F^{\Pi}: K\right]=3$ is odd, by Corollary $5.2, B$ has a $K$-rational point.

(b2) $\mathfrak{G} \cong D_{2 n}, n>2$. The function field of $X / \operatorname{Aut}(X)$ equals the subfield of $F(X)$ fixed by $\mathfrak{G}_{D_{2 n}}$ acting by fractional linear transformations. Then $t:=$ $x^{n}+x^{-n}$ is fixed by $\mathfrak{G}_{D_{2 n}}$ and is a rational function of degree $2 n$ in $x$, so the function field of $X / \operatorname{Aut}(X)$ equals $F(t)$. Therefore we use $t$ as coordinate on $X / \operatorname{Aut}(X)$. The map $\rho: X \rightarrow X / \operatorname{Aut}(X)$ is given by $(x, y) \mapsto\left(x^{n}+x^{-n}\right)$. Let $\sigma \in \Gamma$. By Lemmas 4.2 and 3.3, $\varphi_{\sigma}: X \rightarrow{ }^{\sigma} X$ is given by $(M, e)$ where $\bar{M} \in D_{4 n}$. Then the map $\rho^{\sigma} \varphi_{\sigma}: X \rightarrow{ }^{\sigma} X / \operatorname{Aut}\left({ }^{\sigma} X\right)$ is given by $(x, y) \mapsto$ $\pm\left(x^{n}+x^{-n}\right)$. So $\sigma^{*}(t)= \pm t$. The curve $B$ corresponds to the fixed field of $F(t)$ under $\Gamma^{*}$. Then $t=0$ corresponds to a point $P \in B(K)$.

(c) $\mathfrak{G} \cong A_{4}$. The element $t^{\prime}:=x^{2}+x^{-2}$ is fixed by the normal subgroup $\mathfrak{G}_{D_{4}}$. From (c), we see that the element

$$
t:=\frac{1}{4} t^{\prime}\left(\frac{2 t^{\prime}-12}{t^{\prime}+2}\right)\left(\frac{2 t^{\prime}+12}{-t^{\prime}+2}\right)=\frac{x^{12}-33 x^{8}-33 x^{4}+1}{-x^{10}+2 x^{6}-x^{2}}
$$

is fixed by $\mathfrak{G}_{A_{4}}$ and is a rational function of degree 12 in $x$. So the function field of $X / \operatorname{Aut}(X)$ equals $F(t)$. We use $t$ as coordinate on $X / \operatorname{Aut}(X)$. The 
map $\rho: X \rightarrow X / \operatorname{Aut}(X)$ is given by

$$
(x, y) \mapsto\left(x^{12}-33 x^{8}-33 x^{4}+1\right) /\left(-x^{10}+2 x^{6}-x^{2}\right) .
$$

Let $\sigma \in \Gamma$. By Lemmas 4.2 and 3.3, $\varphi_{\sigma}: X \rightarrow{ }^{\sigma} X$ is given by $(M, e)$ where $\bar{M} \in \mathfrak{G}_{S_{4}}$. A computation shows that $\sigma^{*}(t)= \pm t$. Then $t=0$ corresponds to a point $P \in B(K)$.

(d) $\mathfrak{G} \cong S_{4}$. By Lemma $3.3, N(\mathfrak{G})=\mathfrak{G}$. So by Corollary $4.4, X$ can be defined over $K$.

(e) $\mathfrak{G} \cong A_{5}$. By Lemma $3.3, N(\mathfrak{G})=\mathfrak{G}$. So by Corollary $4.4, X$ can be defined over $K$.

(f) $\mathfrak{G}=\mathfrak{G}_{\beta, A}$. Let $d$ be the order of $\beta$ and let $t=g(x):=\prod_{\alpha \in A}(x-\alpha)^{d}$. Then $t$ is a rational function of degree $|\mathfrak{G}|$ fixed by $\mathfrak{G}_{\beta, A}$ acting by fractional linear transformations. So the function field of $X / \operatorname{Aut}(X)$ equals $F(t)$. We use $t$ as a coordinate function of $X / \operatorname{Aut}(X)$. Let $\sigma \in \Gamma$. By Lemma 4.2, $\varphi_{\sigma}: X \rightarrow{ }^{\sigma} X$ is given by $(M, e)$ where $M$ is an upper diagonal matrix. So $\sigma^{*}(t)=g^{\sigma}(a x+b)$ for some $a \neq 0$ and $b$. Let $P$ be the point of $X / \operatorname{Aut}(X)$ corresponding to $x=\infty$. Then since $g^{\sigma}(a \infty+b)=g(\infty), P$ corresponds to a point in $B(K)$.

(g) $\mathfrak{G}=\mathrm{PSL}_{2}\left(\mathbb{F}_{q}\right)$. It can be deduced from Theorem 6.21 on page 409 of [11] that $\mathrm{PSL}_{2}\left(\mathbb{F}_{q}\right)$ is generated by the image in $\mathrm{PGL}_{2}(F)$ of the following matrices

$$
\left\{\left(\begin{array}{cc}
0 & -1 \\
1 & 0
\end{array}\right),\left(\begin{array}{cc}
1 & a \\
0 & 1
\end{array}\right): a \in \mathbb{F}_{p^{r}}\right\} \text {. }
$$

Let

$$
g(x)=\frac{\left(\left(x^{q}-x\right)^{q-1}+1\right)^{\frac{q+1}{2}}}{\left(x^{q}-x\right)^{\frac{q^{2}-q}{2}}} .
$$

One can verify that $g(-1 / x)=g(x)$ and $g(x+a)=g(x)$ for all $a \in \mathbb{F}_{p^{r}}$. Since $g$ is a rational function of $x$ of degree $\frac{q^{3}-q}{2}=\left|\mathrm{PSL}_{2}\left(\mathbb{F}_{q}\right)\right|$, the function field of $X / \operatorname{Aut}(X)$ is $F(t)$ where $t=g(x)$. We use $t$ as a coordinate function on $X / \operatorname{Aut}(X)$. The map $\rho: X \rightarrow X / \operatorname{Aut}(X)$ is given by

$$
(x, y) \mapsto \frac{\left(\left(x^{q}-x\right)^{q-1}+1\right)^{\frac{q+1}{2}}}{\left(x^{q}-x\right)^{\frac{q^{2}-q}{2}}} .
$$

Let $\sigma \in \Gamma$. By Lemmas 4.2 and 3.3, $\varphi_{\sigma}: X \rightarrow{ }^{\sigma} X$ is given by $(M, e)$ where $\bar{M} \in \mathrm{PGL}_{2}\left(\mathbb{F}_{q}\right)$. A computation shows that $\sigma^{*}(t)= \pm t$. Then $t=0$ corresponds to a point $P \in B(K)$.

(h) $\mathfrak{G}=\mathrm{PGL}_{2}\left(\mathbb{F}_{q}\right)$. By Lemma $3.3, N(\mathfrak{G})=\mathfrak{G}$. So by Corollary $4.4, X$ can be defined over $K$.

Theorem 5.4. Let $K$ be a field of characteristic not equal to 2 , let $X$ be a hyperelliptic curve over $K$ and let $\mathfrak{G}=\operatorname{Aut}(X) /\langle\iota\rangle$ where $\iota$ is the hyperelliptic involution of $X$. Suppose that $\mathfrak{G}$ is not cyclic or that $\mathfrak{G}$ is cyclic of order divisible by the characteristic of $F$. Then $X$ is definable over its field of moduli.

Proof. This follows from Theorem 5.3 and Theorem 2.7. 


\section{Hyperelliptic curves not definable over their fields of moduli}

The first examples of curves not definable over their fields of moduli were discovered by Shimura. These curves are hyperelliptic $\mathbb{C}$-curves with automorphism groups generated by their hyperelliptic involutions and are given on page 177 of [10].

Theorem 5.4 is the best possible in the sense that the hypothesis cannot be weakened: for all $n>1$ we construct a hyperelliptic curve $X$ with $\operatorname{Aut}(X) /\langle\iota\rangle \cong \mathbb{Z} / n \mathbb{Z}$ and of field of moduli $\mathbb{R}$ but not definable over $\mathbb{R}$.

Suppose $n, m \in \mathbb{Z}_{>1}$. Assume that $m$ is odd. For any $z \in \mathbb{C}$ let $z^{c}$ be the complex conjugate of $z$ and let $|z|$ be the norm of $z$. Consider the polynomial $f(x) \in \mathbb{C}[x]$ given by

$$
f(x):=\prod_{1 \leq i \leq m}\left(x^{n}-a_{i}\right)\left(x^{n}+1 / a_{i}^{c}\right),
$$

with $\left|a_{i}\right| \neq\left|a_{j}\right|$ and $a_{i} / a_{i}^{c} \neq a_{j} / a_{j}^{c}$ if $i \neq j$ and $\left|a_{i}\right| \neq\left|1 / a_{j}\right|$ for all $j$. Assume that the constant term of $f$ is -1 . Assume also that for any two zeros $P$ and $Q$ of $f$ we have $P \neq(-2 \pm \sqrt{3}) Q$. (Such polynomials exist. For example take

$$
f(x)=\prod_{1 \leq l \leq m}\left(x^{n}-(l+1) \kappa^{l}\right)\left(x^{n}+(l+1)^{-1} \kappa^{l}\right)
$$

where $\kappa$ is a primitive $m^{t h}$ root of unity.)

Lemma 6.1. Following the above notation, let $X$ be the hyperelliptic curve over $\mathbb{C}$ given by $y^{2}=f(x)$. Let $\iota$ be the hyperelliptic involution of $X$ and let $\nu$ be the automorphism of $X$ defined by $\nu(x, y)=(\zeta x, y)$, where $\zeta$ is a primitive $n^{\text {th }}$ root of unity. Then $\operatorname{Aut}(X)=\langle\iota\rangle \oplus\langle\nu\rangle$.

Proof. Let $\mathfrak{G}=\operatorname{Aut}(X) /\langle\iota\rangle$. Suppose that $\mathfrak{G}$ is not cyclic of order $n$. By Lemma 3.1, $\mathfrak{G} \cong C_{n^{\prime}}, D_{2 n^{\prime}}, A_{4}, S_{4}$, or $A_{5}$ where $n^{\prime}>n$ in the first case and $n^{\prime} \geq n$ in the second case. Let $\bar{\nu}$ be the image of $\nu$ under the quotient map $\operatorname{Aut}(X) \rightarrow \mathfrak{G}$. So $\bar{\nu}$ is the image in $\mathrm{PGL}_{2}(\mathbb{C})$ of the matrix

$$
\left(\begin{array}{ll}
\zeta & 0 \\
0 & 1
\end{array}\right)
$$

Let $\langle\bar{\nu}\rangle$ be the subgroup of $\mathfrak{G}$ generated by $\bar{\nu}$.

Using the structure of the abstract groups $C_{n^{\prime}}, D_{2 n^{\prime}}, A_{4}, S_{4}$, and $A_{5}$, we can deduce the following. If $n=2$, then $\langle\bar{\nu}\rangle$ is contained in a subgroup of $\mathfrak{G}$ isomorphic to $C_{n^{\prime}}$ with $n^{\prime}>2$, or $\langle\bar{\nu}\rangle$ is contained in a subgroup of $\mathfrak{G}$ isomorphic to $D_{4}$, or $\mathfrak{G} \cong D_{2 n^{\prime}}$ with $n^{\prime}>1$. If $n=3$, then either $\mathfrak{G} \cong C_{n^{\prime}}$ with $n^{\prime}>3$, or $\langle\bar{\nu}\rangle$ is contained in a subgroup of $\mathfrak{G}$ isomorphic to $D_{6}$ or $A_{4}$. If $n$ is equal to 4 or 5 , then $\mathfrak{G} \cong C_{n^{\prime}}$ with $n^{\prime}>n$ or $\langle\bar{\nu}\rangle$ is contained in a subgroup of $\mathfrak{G}$ isomorphic to $D_{2 n}$. If $n>5$, then either $\mathfrak{G} \cong C_{n^{\prime}}$ with $n^{\prime}>n$ or $\mathfrak{G} \cong D_{2 n^{\prime}}$ with $n^{\prime} \geq n$.

For each $P \in \mathbb{C} \cup\{\infty\}$ and $g:=\left[\begin{array}{ll}a & b \\ c & d\end{array}\right] \in \mathrm{PGL}_{2}(\mathbb{C})$, let $g(P)=\frac{a P+b}{c P+d}$. Let $P_{1} \ldots P_{r}$ be the zeros of $f$. If $g \in \mathrm{PGL}_{2}(\mathbb{C})$ lifts to an automorphism of $X$, then

$$
\left\{P_{1}, \ldots, P_{r}\right\}=\left\{g\left(P_{1}\right), \ldots, g\left(P_{r}\right)\right\} .
$$

The conditions $\left|a_{i}\right| \neq\left|a_{j}\right|$ if $i \neq j$ and $\left|a_{i}\right| \neq\left|1 / a_{j}\right|$ for all $j$ guarantee the following. Let $P$ be a zero of $f$ with $|P|=\lambda$. Then a zero of $f$ has norm $\lambda$ if and only if it is a 
zero of $x^{n}-P^{n}$. In particular, if for some $a \in \mathbb{C} x^{n}-a$ divides $f(x)$ and $|a|=\left|a_{i}\right|$ or $|a|=\left|1 / a_{i}\right|$ for some $i$ then $a=a_{i}$ or $a=-1 / a_{i}^{c}$ respectively.

First suppose that $\langle\bar{\nu}\rangle$ is contained in a cyclic subgroup $\mathfrak{G}^{\prime}$ of $\mathfrak{G}$ of order $n^{\prime}>n$. Since the only elements of order larger than 2 in $\mathrm{PGL}_{2}(\mathbb{C})$ that commute with $\bar{\nu}$ are the images of diagonal matrices and since $\mathfrak{G}^{\prime}$ has order $n^{\prime}$, a generator for $\mathfrak{G}^{\prime}$ is given by

$$
\left[\begin{array}{ll}
\zeta^{\prime} & 0 \\
0 & 1
\end{array}\right]
$$

where $\zeta^{\prime}$ is a primitive $\left(n^{\prime}\right)^{t h}$ root of unity. Since this element lifts to an automorphism of $X$ we must have

$$
\prod_{1 \leq i \leq m}\left(x^{n}-a_{i}\right)\left(x^{n}+1 / a_{i}^{c}\right)=\prod_{1 \leq i \leq m}\left(x^{n}-\left(\zeta^{\prime}\right)^{n} a_{i}\right)\left(x^{n}+\left(\zeta^{\prime}\right)^{n} / a_{i}^{c}\right) .
$$

This is a contradiction since $\left|\left(\zeta^{\prime}\right)^{n} a_{i}\right|=\left|a_{i}\right|$ for all $i$ and by assumption $\left(\zeta^{\prime}\right)^{n} \neq 1$.

Now suppose that either $n>2$ and $\langle\bar{\nu}\rangle$ is contained in a dihedral subgroup $\mathfrak{G}^{\prime}$ of $\mathfrak{G}$ or $n=2$ and $\langle\bar{\nu}\rangle$ is contained in a subgroup $\mathfrak{G}^{\prime}$ of $\mathfrak{G}$ isomorphic to $D_{4}$. Then there exists an element $\bar{u} \in \mathfrak{G}^{\prime}$ of order 2 with $\bar{u} \bar{\nu} \bar{u}=\bar{\nu}^{-1}$. A computation shows that $\bar{u}$ must be an element of the form

$$
\left[\begin{array}{ll}
0 & \alpha \\
1 & 0
\end{array}\right]
$$

for some $\alpha \in \mathbb{C}^{\times}$. Then we must have

$$
\prod_{1 \leq i \leq m}\left(x^{n}-a_{i}\right)\left(x^{n}+1 / a_{i}^{c}\right)=\prod_{1 \leq i \leq m}\left(x^{n}-(\alpha)^{n} / a_{i}\right)\left(x^{n}+(\alpha)^{n} a_{i}^{c}\right) .
$$

Since the constant term of $f$ is $-1, \alpha$ must be a root of unity. Since $\left|a_{i}\right|=\left|(\alpha)^{n} a_{i}^{c}\right|$ for all $i$, we must have $a_{i}=(\alpha)^{n} a_{i}^{c}$ for all $i$. This contradicts the condition $a_{i} / a_{i}^{c} \neq a_{j} / a_{j}^{c}$ if $i \neq j$.

Now suppose that $n=2$ and that $\mathfrak{G} \cong D_{2 n^{\prime}}$ with $n^{\prime}>1$ and odd. Since $\mathfrak{G}$ is conjugate to $\mathfrak{G}_{D_{2 n^{\prime}}}$, there exists an element $\bar{M}$ of $\mathrm{PGL}_{2}(\mathbb{C})$ with $\bar{M} \mathfrak{G}(\bar{M})^{-1}=\mathfrak{G}_{D_{2 n^{\prime}}}$. Suppose that $\bar{M}$ is the image in $\mathrm{PGL}_{2}(\mathbb{C})$ of the matrix

$$
M:=\left(\begin{array}{ll}
a & b \\
c & d
\end{array}\right) .
$$

Let

$$
h(x):=(-c x+a)^{4 m} f\left(\frac{d x-b}{-c x+a}\right) \in \mathbb{C}[x] .
$$

Let $Y$ be the hyperelliptic curve given by $y^{2}=h(x)$. Using the notation in Proposition 4.1, there exists $e \in \mathbb{C}^{\times}$such that $(M, e)$ gives an isomorphism $\varphi: X \rightarrow Y$. Let $\iota^{\prime}$ be the hyperelliptic involution of $Y$. We see that $\operatorname{Aut}(Y) /\left\langle\iota^{\prime}\right\rangle=\mathfrak{G}_{2 n^{\prime}}$. The map $\mu$ defined by

$$
\mu(x, y)=\left((i x)^{-1}, i x^{-n m} y\right),
$$

is an isomorphism between the curve $X$ and the complex conjugate curve ${ }^{c} X$. So the map $\varphi^{c} \mu \varphi^{-1}$ is an isomorphism from $Y$ to ${ }^{c} Y$. By Lemmas 4.2 and 3.3, the image in $\mathrm{PGL}_{2}(\mathbb{C})$ of the matrix

$$
\left(\begin{array}{ll}
a^{c} & b^{c} \\
c^{c} & d^{c}
\end{array}\right)\left(\begin{array}{ll}
0 & 1 \\
i & 0
\end{array}\right)\left(\begin{array}{cc}
d & -b \\
-c & a
\end{array}\right)=\left(\begin{array}{ll}
b^{c} d i-a^{c} c & a a^{c}-b b^{c} i \\
d d^{c} i-c c^{c} & a c^{c}-b d^{c} i
\end{array}\right)
$$


is in $N\left(\mathfrak{G}_{D_{2 n^{\prime}}}\right)=\mathfrak{G}_{D_{4 n^{\prime}}}$. Since $a a^{c}-b b^{c} i \neq 0$, we must have $b^{c} d i=a^{c} c$ and $a c^{c}=b d^{c} i$. Taking the complex conjugate of both sides of the first equation, we see that either $a=d=0$ or $b=c=0$. Then $\frac{b b^{c}}{c c^{c}} i$ or $\frac{a a^{c}}{d d^{c}} i$ is a $\left(2 n^{\prime}\right)^{t h}$ root of unity. Since $n^{\prime}$ is odd, this is a contradiction.

Now suppose that $n=3$ and $\langle\bar{\nu}\rangle$ is contained in a subgroup $\mathfrak{G}^{\prime}$ of $\mathfrak{G}$ isomorphic to $A_{4}$. The group $\mathfrak{G}^{\prime}$ acts on the hyperelliptic branch points of $X$ by fractional linear transformation. Since $m$ is odd, the number of hyperelliptic branch points of $X$ is congruent to $6(\bmod 12)$. So by Proposition 2.1 and Lemma 2.2 of [2], there is a zero $P$ of $f$ whose orbit $\mathfrak{O}$ under the action of $\mathfrak{G}^{\prime}$ has six elements. Then there exists a zero $Q$ of $f(x)$ such that $\mathfrak{O}=\left\{P, \zeta P, \zeta^{2} P, Q, \zeta Q, \zeta^{2} Q\right\}$. By $\S 73$ of [12], there is exactly one orbit $\mathfrak{O}^{\prime}:=\{0, \infty, \pm 1, \pm i\}$ of $\mathbb{C} \cup\{\infty\}$ under the action of $\mathfrak{G}_{A_{4}}$ of size 6 . One can verify that for any element $g \in \mathfrak{G}_{A_{4}}$ of order 3 , there exists an element $h \in \mathfrak{G}_{A_{4}}$ of order 2 and $P^{\prime}, Q^{\prime} \in \mathfrak{O}^{\prime}$ such that $\mathfrak{O}^{\prime}=\left\{P^{\prime}, g\left(P^{\prime}\right), g^{2}\left(P^{\prime}\right), Q^{\prime}, g\left(Q^{\prime}\right), g^{2}\left(Q^{\prime}\right)\right\}, h\left(P^{\prime}\right)=P^{\prime}$, $h\left(Q^{\prime}\right)=Q^{\prime}, h\left(g\left(P^{\prime}\right)\right)=g\left(Q^{\prime}\right)$, and $h\left(g^{2}\left(P^{\prime}\right)\right)=g^{2}\left(Q^{\prime}\right)$.

Since $\mathfrak{G}^{\prime}$ is conjugate to $\mathfrak{G}_{A_{4}}$, there exists an element $\bar{u} \in \mathfrak{G}^{\prime}$ of order 2 such that $\bar{u}\left(\zeta^{i} P\right)=\zeta^{i} P, \bar{u}\left(\zeta^{j} Q\right)=\zeta^{j} Q, \bar{u}\left(\zeta^{i+1} P\right)=\zeta^{j+1} Q$, and $\bar{u}\left(\zeta^{i+2} P\right)=\zeta^{j+2} Q$ for some $i$ and $j$. Replacing $P$ with $\zeta^{i} P$ and $Q$ with $\zeta^{j} Q$ we may assume that $\bar{u}(P)=P$, $\bar{u}(Q)=Q, \bar{u}(\zeta P)=\zeta Q$, and $\bar{u}\left(\zeta^{2} P\right)=\zeta^{2} Q$. Any element of order 2 in $\mathrm{PGL}_{2}(\mathbb{C})$ is conjugate to

$$
\left[\begin{array}{cc}
-1 & 0 \\
0 & 1
\end{array}\right]
$$

and so fixes exactly 2 points of $\mathbb{C} \cup\{\infty\}$ and is the image of a matrix with trace 0 . Since $\bar{u}$ does not fix $\infty, \bar{u}$ is the image in $\mathrm{PGL}_{2}(\mathbb{C})$ of a matrix of the form

$$
\left(\begin{array}{cc}
a & b \\
1 & -a
\end{array}\right) \text {. }
$$

Solving

$$
P=\frac{a P+b}{P-a}
$$

and

$$
Q=\frac{a Q+b}{Q-a}
$$

for $a$ and $b$ we see that $\bar{u}$ is the image in $\mathrm{PGL}_{2}(\mathbb{C})$ of

$$
\left(\begin{array}{cc}
\frac{P+Q}{2} & -P Q \\
1 & -\frac{P+Q}{2}
\end{array}\right) \text {. }
$$

Since

$$
\zeta Q=\frac{\zeta P\left(\frac{P+Q}{2}\right)-P Q}{\zeta P-\frac{P+Q}{2}},
$$

we have

$$
Q^{2}+4 P Q+P^{2}=0
$$

So $P=(-2 \pm \sqrt{3}) Q$. This is a contradiction.

Therefore the image of $\nu$ under the quotient map $\operatorname{Aut}(X) \rightarrow \mathfrak{G}$ generates all of $\mathfrak{G}$. Since $\iota$ and $\nu$ commute and generate a subgroup of order $2 n$ we have $\operatorname{Aut}(X)=\langle\iota\rangle \oplus\langle\nu\rangle$. 
Proposition 6.2. Let $X$ be as in Lemma 6.1. The field of moduli of $X$ relative to the extension $\mathbb{C} / \mathbb{R}$ is $\mathbb{R}$ and is not a field of definition for $X$.

Proof. By Lemma 6.1, Aut $(X)=\langle\iota\rangle \oplus\langle\nu\rangle$ where $\iota$ is the hyperelliptic involution of $X$, and $\nu(x, y)=(\zeta x, y)$ where $\zeta$ is a primitive $n^{t h}$ root of unity. The map $\mu$ defined by

$$
\mu(x, y)=\left((\omega x)^{-1}, i x^{-n m} y\right)
$$

where $\omega^{n}=-1$, is an isomorphism between the curve $X$ and the complex conjugate curve ${ }^{c} X$. Any isomorphism $X \rightarrow{ }^{c} X$ is given by $\mu \nu^{k}$, or $\mu \iota \nu^{k}$ for some $0 \leq k \leq n-1$. We have $\mu \iota=\iota \mu$,

$$
\mu \nu(x, y)=\left((\omega \zeta x)^{-1}, i(\zeta x)^{-n m} y\right)=\nu^{c} \mu(x, y),
$$

and

$$
\mu^{c} \mu(x, y)=\left(\left(\omega^{-1}(\omega x)^{-1}\right)^{-1},-i(\omega x)^{n m}\left(i x^{-n m} y\right)\right)=\left(\omega^{2} x,-y\right)=\nu^{l} \iota(x, y)
$$

for some $l$. Then

$$
\left(\mu \nu^{k}\right)^{c} \mu \nu^{k}=\mu^{c} \nu^{-k} \mu \nu^{k}=\mu^{c} \mu \nu^{2 k}=\iota \nu^{2 k+l} \neq I d
$$

and

$$
\left(\mu \iota \nu^{k}\right)^{c} \mu \iota \nu^{k}=\mu^{c} \iota \nu^{-k} \mu \iota \nu^{k}=\mu^{c} \mu \nu^{2 k}=\iota \nu^{2 k+l} \neq I d .
$$

Therefore Weil's cocycle condition from Theorem 2.9 does not hold. So $X$ cannot be defined over $\mathbb{R}$.

\section{Acknowledgements}

The author learned of the conjecture of [9] from Bjorn Poonen, and thanks him for comments on an early draft of this paper. The author was partially supported by NSF grant DMS-0301280 of Bjorn Poonen. The author also thanks the anonymous referee for several helpful comments.

\section{References}

[1] M. H. Baker, E. González-Jiménez, J. Gonzáles, and B. Poonen, Finiteness results for modular curves of genus at least 2..

[2] R. Brandt and H. Stichtenoth, Die Automorphismengruppen hyperelliptischer Kurven, Manuscripta Math. 55 (1986), no. 1, 83-92.

[3] E. Bujalance and P. Turbek, Asymmetric and pseudo-symmetric hyperelliptic surfaces, Manuscripta Math. 108 (2002), no. 1, 1-11.

[4] P. Dèbes and J.-C. Douai, Algebraic covers: field of moduli versus field of definition, Ann. Sci. École Norm. Sup. (4) 30 (1997), no. 3, 303-338.

[5] P. Dèbes and M. Emsalem, On fields of moduli of curves, J. Algebra 211 (1999), no. 1, 42-56.

[6] J. Q. G. Cardona, Field of moduli and field of definition for curves of genus 2, in T. Shaska, editor, Computational aspects of algebraic curves, Vol. 13 of Lecture Notes Series on Computing, 71-83, World Sci. Publishing, Hackensack, NJ (2005).

[7] J. Gutierrez and T. Shaska, Hyperelliptic curves with extra involutions, LMS J. Comput. Math. 8 (2005) 102-115 (electronic).

[8] B. Huggins, Fields of moduli and fields of definition of curves, Ph.D. thesis, University of California, Berkeley, Berkeley, California (2005). Available at http://arxiv.org/abs/math.NT/0610247.

[9] T. Shaska, Computational Aspects of Hyperelliptic Curves, in Computer mathematics. Proceedings of the sixth Asian symposium (ASCM 2003), Beijing, China, April 17-19, 2003, Vol. 10 of Lecture Notes Series on Computing, 248-257, World Sci. Publishing, River Edge, NJ (2003). 
[10] G. Shimura, On the field of rationality for an abelian variety, Nagoya Math. J. 45 (1972) $167-178$.

[11] M. Suzuki, Group theory. I, Vol. 247 of Grundlehren der Mathematischen Wissenschaften [Fundamental Principles of Mathematical Sciences], Springer-Verlag, Berlin (1982), ISBN 3-54010915-3. Translated from the Japanese by the author.

[12] H. Weber, Lehrbuch der Algebra, Vol. II, Vieweg, Braunschweig, second edition (1899).

[13] A. Weil, The field of definition of a variety, Amer. J. Math. 78 (1956) 509-524.

Bloomberg LP, 731 Lexington Ave, New York, NY 10022

E-mail address: bhuggins@math.berkeley.edu 